\title{
Crecimiento postnatal del recién nacido de muy bajo peso: II antropometría a un año plazo, estudio longitudinal
}

\author{
Dra. Mafalda Rizzardini P. ${ }^{1}$; Dr. Mario Ferreiro S. ${ }^{1}$; Dr. Lionel Bernier ${ }^{1}$; \\ Sr. Pablo Bernier B. ${ }^{2}$
}

\section{Postnatal growth in very low birth weight infants II}

\begin{abstract}
A prospective study up to one yea of age, was done in 154 newboms with birth weight less than $1,501 \mathrm{~g}$ at a Metropolitan Hospital of Santiago, Chile, between 1983 and 1987. The patients wete divided in groups A: 44 patients with birth weight less than $1,001 \mathrm{~g} ; \mathrm{B}: 68$ patients with birth weights from 1,000 to $1,250 \mathrm{~g}$ and $\mathrm{C} ; 48$ patients with birth weight 1,251 to $1,500 \mathrm{~g}$. At age one year weight of group A patients was $6,724 \pm 804 \mathrm{~g} ; \mathrm{group}$ B $7,782 \pm 927 \mathrm{~g}$ and group C 7,941 $+903 \mathrm{~g}$. Weight and height of group $\mathrm{A}$ babies at one year of age were below 2 DS of the average weight and height for normal chilen babies of the same socioeconomic status and geografic area with $3,318 \mathrm{~g}$ nean birth weight. Group B and C were between 1 and $2 \mathrm{DS}$. Head circunterence was within normal ranges in groups $B$ and $C$ patients, but in group A patients if was below 2 DS of the average.

(Key words: birth weight, infants, low birth weight, growth.)
\end{abstract}

El crecimiento postnatal del recién nacido de bajo peso $(\leqslant 2.500 \mathrm{~g}$ ) ha sido motivo de muchos estudios. La mayor parte de ellos parecen demostrar que el crecimiento extrauterino es semejante al del feto in tutero, de tal manera que, si se co. rrige la edad postconcepcional, las curvas de cre-

1. Departamento de Pediatrja Hospital R. del Río. Santiago, Chile.

2. Interno de la carrera de Medicina. cimiento de los niños nacidos a término podrían servir para evaluar el estado nutritivo de los de gestaciones más cortas ${ }^{1-3}$. Esto no parece sè aplicable al recién nacido de muy bajo peso (RNMBP), inferior a $1.501 \mathrm{~g}$. Ellos padecen de numerosos trastomos durante las primeras semanas de vida $y$, aunque estén libres de enfermedades graves, a las 40 semanas de edad corregida, el peso está muy por debajo de lo normal ${ }^{4-6}$. 
A pesar de que el aumento significativo de la sobrevida del RNMBP ha promovido gran interés en conocer su evolución a largo plazo, existe muy poca información sobre su crecimiento después de los primeros tres meses de vida, tiempo durante el cual los nifios permanecen hospitalizados y se ensayan distintos tipos de alimentación ${ }^{7-9}$.

En una comunicación anterior ${ }^{10}$ dimos a conocer el crecimiento en las primeras ocho semanas de vida de 126 RNMBP atendidos en el Hospital Roberto del Río entre 1983-87. En esta oportunidad nos referiremos a las medidas antropométricas de esos mismos niños y de otros que se incorporaron más tarde al estudio y que han cumplido, por lo menos, 1 año de edad.

El objeto de esta publicación es entregar una guía para la calificación del estado nutritivo de los niños que al nacer pesan $1.500 \mathrm{~g}$ o menos, basada en los resultados obtenidos en un grupo de recién nacidos de la zona norte de Santiago.

\section{MATERIAL Y METODOS}

Se estudió en forma prospectiva el crecimiento longitudinal de 154 recién nacidos de 660 a $1.500 \mathrm{~g}$ que egresaron de la Unidad de Neonatología del Hospital Roberto del Río entre 1983-1987. Este centro atiende una población urbana de escasos recursos y los niños nacen en una maternidad anexa al hospital pedítrico. Se incluyeron en el estudio sólo neonatos, libres de anomalias congénitas, otras enfermedades graves o signos de malnutrición intrauterina. No se excluyeron los con trastornos propios de su inmadurez (sindrome de dificultad respiratoria, crisis de apnea, ductus persistente, exigiéndose sólo que los niños seleccionados sobrevivieran hasta el alta del hospital.

Los métodos usados para el control antropométrico fueron los de Patrí $y$ colaboradores ${ }^{1}$ y las curvas de crecimiento para nirios normales de la zona norte de Santiago, de los mismos autores, se usaron con fïnes de comparación.

Para el análisis del material se dividieron los sujetos en tres grupos de acuerdo al peso de nacimiento. E1 grupo A incluyó 44 recién nacidos de 1.000 g a menos; el grupo B, 68 de 1.001 a $1.250 \mathrm{~g}$, y el grupo $\mathrm{C}, 42$ pacientes de 1.251 a $1.500 \mathrm{~g}$. En la tabla 1 se anotan las caracter ísticas del material.

\section{RESULTADOS}

Despuès del descenso inicial el peso aumenta lentamente durante los dos primeros meses, especialmente el de los recién nacidos de menos de $1.001 \mathrm{~g}$. Más tarde el ascenso ponderal mensual supera al esperado para un recién nacido a término. Al año los niños del grupo A pesan

Tabk 1

Características clínicas al nacer de 154 tecién nacidos de muy bajo peso, según grupo

\begin{tabular}{|c|c|c|c|}
\hline GRUPO & $(\leqslant 1.000 \mathrm{~g})$ & $\frac{\mathrm{B}}{(1.001 \cdot 1.250 \mathrm{~g})}$ & $\begin{array}{c}\mathrm{C} \\
(1.25 \perp-[.500 \mathrm{~g})\end{array}$ \\
\hline NUMERO & 44 & 68 & 42 \\
\hline \multicolumn{4}{|l|}{ EDAD } \\
\hline \multicolumn{4}{|l|}{ GEST ACIONAL } \\
\hline$\vec{X} \pm D E$ semanas & $27,1 \pm 1,0$ & $28,5 \pm 1,1$ & $30,3 \pm 1,2$ \\
\hline Rango & $(24-29)$ & $(27 \cdot 3 \mathbf{I})$ & $(28-33)$ \\
\hline \multicolumn{4}{|l|}{ PESO DE } \\
\hline \multicolumn{4}{|l|}{ NACIMIENTO } \\
\hline$\overline{\mathbf{X}} \pm \mathrm{DE} \mathbf{g}$ & $918 \pm 87,7$ & $1.137 \pm 63,6$ & $1.386 \pm 75,2$ \\
\hline Rango & $(660-1.000)$ & $(1.020-1.250)$ & $(1.260-1.500)$ \\
\hline \multicolumn{4}{|l|}{ TALLA } \\
\hline $\bar{X} \pm D E \mathrm{~cm}$ & $35,0 \pm 1,6$ & $37,2 \pm 1,2$ & $39,5 \pm 1,4$ \\
\hline Rango & $(31$ - 38) & $(33.5-40)$ & $(36-42)$ \\
\hline \multicolumn{4}{|l|}{ PERMETRO } \\
\hline \multicolumn{4}{|l|}{ CEFALICO } \\
\hline$\overline{\mathrm{X}} \pm \mathrm{DE} \mathrm{cm}$ & $24,7 \pm 1,2$ & $26.3 \pm 1,2$ & $27,8 \pm 1,4$ \\
\hline Rango & $(22-28)$ & $(24-28,5)$ & $(25,2-30)$ \\
\hline
\end{tabular}


en promedio $6.724 \mathrm{~g} \pm 804$; los del grupo $\mathrm{B}$, $7.782 \mathrm{~g} \pm 927, y$ los del grupo C, $7.941 \mathrm{~g} \pm 903$. Los incrementos anuales alcanzan a 5.847 , 6.641 y $6.651 \mathrm{~g}$ en los tres grupos, respectiva. mente (tabla 2).

La talla tiene un incremento lineal mayor que el de los $\mathrm{RN}$ a término, excepto en el primer mes de vida. Al cumplir 12 meses los ninos del grupo A miden un promedio de $66,7 \pm 2,3 \mathrm{~cm}$; los del grupo B, $69,1 \pm 2,5 \mathrm{~cm}, y$ los del grupo C, $70,5 \pm 2,9 \mathrm{~cm}$, con incrementos anuales totales de 31,$7 ; 31,9$ y $31 \mathrm{~cm}$ en los tres grupos, respectivamente (tabla 3 ).

El aumento del perímetro cefálico es casi igual o superior al de los recién nacidos a término desde el primer mes de vjda. Al año esta medida antropométrica alcanza a $42,9 \pm 1,5 \mathrm{~cm}$ en el grupo $A, 44,8 \pm 1,2 \mathrm{~cm}$ en el grupo B, $y$ a 44,7 $\pm 1,4 \mathrm{~cm}$ en el grupo C. Los incrementos totales en el año a 18,$2 ; 18,8, y 16,9$, en los ninos de los grupos A, B y C, respectivamente (tabla 4).

Tabla 2

Peso promedio \pm 1 DE (gramos) durante los primeros meses de vida en recién nacidos de muy bajo peso

\begin{tabular}{|c|c|c|c|}
\hline Grupo & $\stackrel{\mathrm{A}}{(\leqslant 1.000 \mathrm{~g})}$ & $(1.001-1.250 \mathrm{~g})$ & $(1.251-1.500 \mathrm{~g})$ \\
\hline \multicolumn{4}{|l|}{ Meses } \\
\hline 1 & $998 \pm 153,2$ & $1.284 \pm 156,9$ & $1.573 \pm 215,8$ \\
\hline 2 & $1.463 \pm 237,4$ & $1.832 \pm 260,7$ & $2.430 \pm 575,6$ \\
\hline 3 & $2.263 \pm 324,3$ & $2.842 \pm 481,1$ & $3.398 \pm 625,9$ \\
\hline 6 & $4.206 \pm 647,2$ & $5,198 \pm 594,9$ & $5.416 \pm 943,3$ \\
\hline 9 & $5.856 \pm 847,5$ & $6.687 \pm 824,5$ & $6.854 \pm 664,3$ \\
\hline 12 & $6.724 \pm 804,9$ & $7.782 \pm 927,1$ & $7.941 \pm 900,3$ \\
\hline $\begin{array}{l}\text { Incremento total } \\
\text { anual (g) }\end{array}$ & 5.847 & 6.641 & 6.651 \\
\hline $\begin{array}{l}\text { Incremento porcentual } \\
\text { al afío según Patri }\end{array}$ & $95,7 \%$ & $109 \%$ & $108 \%$ \\
\hline
\end{tabular}

Tabla 3

Talla promedio $\pm 1 \mathrm{DE}(\mathrm{cm})$ đurante los primeros meses de vida en tecién nacidos de muy bajo peso

\begin{tabular}{|c|c|c|c|c|}
\hline Grupo & $(\leqslant 1.000 \mathrm{~g})$ & $(1.001-1.250 \mathrm{~g})$ & $(1.251$ & $\begin{array}{l}\mathrm{C} \\
1 \cdot 1.500 \mathrm{~g})\end{array}$ \\
\hline \multicolumn{5}{|l|}{ Meses } \\
\hline 1 & $37,1 \pm 2,0$ & $39,8 \pm 1,0$ & 41,8 & $\pm 1,9$ \\
\hline 2 & $40,5 \pm 2,2$ & $43,3 \pm 1,8$ & 45,7 & $\pm 2,4$ \\
\hline 3 & $44,5 \pm 2,9$ & $47,7 \pm 1,5$ & 49,3 & $\pm 2,4$ \\
\hline 6 & $54,1 \pm 2,0$ & $58,1 \pm 2,1$ & 59,4 & $\pm 2,4$ \\
\hline 9 & $62,0 \pm 2,0$ & $64,3 \pm 1,9$ & 65,3 & $\pm 1,9$ \\
\hline 12 & $66,7 \pm 2,3$ & $69,1 \pm 2,5$ & 70,5 & $\pm 2,9$ \\
\hline $\begin{array}{l}\text { Incremento total } \\
\text { anual }(\mathrm{cm})\end{array}$ & \multicolumn{2}{|l|}{31,7} & \multicolumn{2}{|r|}{31,0} \\
\hline $\begin{array}{l}\text { Incremento porcentual } \\
\text { al año según Patrí }\end{array}$ & $133,2 \%$ & $134,0 \%$ & \multicolumn{2}{|r|}{$130,2 \%$} \\
\hline
\end{tabular}


Tabla 4

Perímetro cefálico promedio $\pm 1 \mathrm{DE}(\mathrm{cm})$ duxante los primeros meses de vida en recién nacidos de muy bajo peso

\begin{tabular}{cccc}
\hline Grupo & A & B & C \\
& $(\leqslant 1.000 \mathrm{~g})$ & $(1.001-1.250 \mathrm{~g})$ & $(1.251 \cdot 1,500$ \\
\hline Meses & & & \\
1 & $26,4 \pm 1,8$ & $29,0 \pm 1,3$ & $29,9 \pm 1,4$ \\
2 & $29,5 \pm 1,5$ & $31,6 \pm 1,9$ & $33,4 \pm 1,8$ \\
3 & $33,1 \pm 2,0$ & $35,3 \pm 1,2$ & $35,9 \pm 1,5$ \\
6 & $38,3 \pm 1,3$ & $39,8 \pm 1,5$ & $40,5 \pm 2,0$ \\
9 & $41,5 \pm 1,5$ & $42,7 \pm 1,2$ & $43,1 \pm 1,1$ \\
12 & $42,9 \pm 1,5$ & $44,8 \pm 1,2$ & $44,7 \pm 1,4$
\end{tabular}

Incremento total

anual $(\mathrm{cm})$

18,2

18,8

16,9

Incremento porcentual

al año según Patıí ${ }^{11}$

$171,7 \%$

$174,5 \%$

$159,4 \%$

\section{DISCUSION}

El déficit de crecimiento postnatal es uno de los más importantes problemas del RNMBP que sobrevive a las numerosas contingencias del periodo neonatal inmediato Para catalogar correctamente el estado nutritivo de estos pacientes, han aparecido algunas curvas de crecimiento en las que se relaciona el peso y la edad postconcepcional. Según algunas, el crecimiento postnatal reproduce el intrauterino, por lo que, corregida la edad de gestación, podrian usarse las curvas de los niños nacidos a término para catalogar el estado nutritivo de los prematuros ${ }^{1-3}$. Esto podría ser aceptable para los que nacen muy cerca de las 40 semanas, pero difícilmente para los productos de gestaciones muy cortas. El importante descenso ponderal inicial y el lento ascenso, durante los dos primeros meses de vida, hacen que a las 40 semanas la mayor parte de ellos tenga un peso bajo el percentil 10, aunque al nacer haya sido catalogado como adecuado para la edad gestacional ${ }^{4-6}$. Por otro lado, la edad intrauterina no siempre es conocida o con. fiable, lo que limita la aplicación en la práctica diaria de las curvas de crecimiento en que se toma en cuenta este dato.

Es muy dificil comparar nuestros resultados con los de otros autores porque los criterios usa. dos para la selección del materjal y para el análi. sis de los datos son diferentes, además el creci. miento a largo plazo está sujeto a una serie de variables inherentes al medio ambiente $y$ a las caracteristicas étnicas. Por estas razones hemos preferido usar, con fines de comparación, las curvas de crecimiento de Patrí y colaboradores ${ }^{11}$, las que incluyen la antropometría de niños na. cidos a término con un peso promedio đe $3.318 \mathrm{~g}$ y pertenecientes al mismo medio socioeconómi. co y área geográfica.

En este material el peso sube muy lenta. mente durante los dos primeros meses de edad, especialmente en los RN de menos de $1.001 \mathrm{~g}$. Estos rifíos aumentan sólo $80 \mathrm{~g}$ en el primer mes de vida contra 699 de los niños normales. Más tarde, aunque los incrementos mensuales superan a los de Patrí, el incremento total al año sólo corresponde al $95,7 \%$ de éstos. A los doce meses de edad postnatal el peso promedio de los RN del grupo A cabe bajo $2 \mathrm{DE}$ en relación con los patrones mencionados (figura 1). Estos resultados coinciden con los de Britton ${ }^{12}$, quien, en $158 \mathrm{RN}$ de 700 a $800 \mathrm{~g}$, encuentra que al año de edad corregida el $57 \%$ está bajo el percentil 3, en peso (bajo $2 \mathrm{DE}$ ).

El incremento ponderal promedio de los niños en estudio, de los grupos B y C, es mayor que el de los del grupo A, correspondiendo al 106 y $103 \%$, respectivamente, según los patrones normales. A los doce meses el peso de estos sujetos cae entre 1 y $2 \mathrm{DE}$ bajo el promedio normal. El peso a esta edad, observado en este estu- 


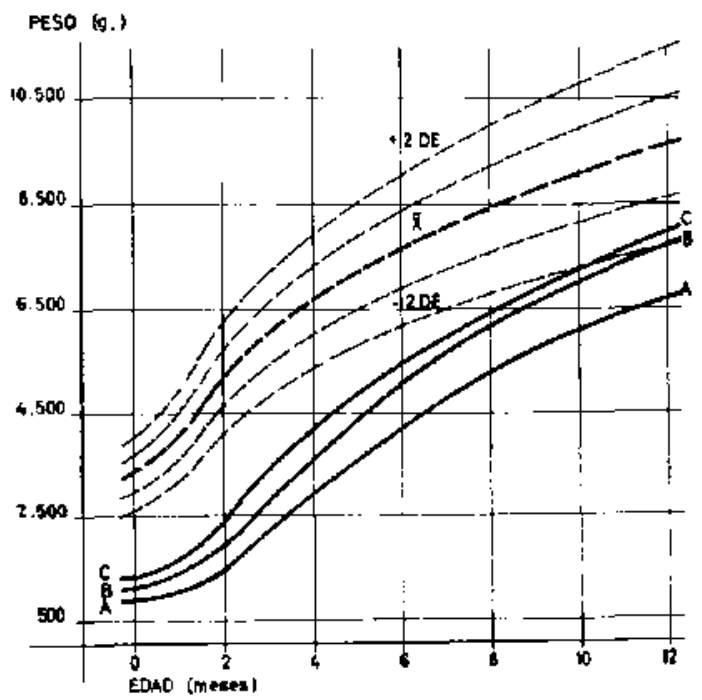

Figura 1: Peso según edad postnatal en tees grupos de recién nacidos de muy bajo peso: grupo A, $\leqslant 1.000 \mathrm{~g}$; grupo B, 1.001-1.250 g; grupo C, 1.250-1.500 g. Comparado con las curvas de crecimiento de recién nacidos nomales según Patrí y col." ".

dio, es muy inferior al comunicado por otros autores ${ }^{4-6,13}$, quienes corrigen la edad. Como éstos no đạ información sobre el factor de corrección, no es posible hacer comparaciones váliđas. Kimble ${ }^{5}$, en cambio, da un factor de corrección de 1,2 meses $y$ sus datos coinciden con los de esta experiencia.

La talla y el tamaño de la cabeza tienen un más rápido incremento que el peso. Al año de edad, ambas medidas antropométricas caen en este estudio bajo $2 \mathrm{DE}$ sólo en el grupo A y se acercan mucho al promedio en los grupos B y C. La mayor parte de los autores $5,6,13,14$ parecen aceptar que la talla y el perímetro cefálico, por tener un crecimiento lineal, desde el nacimiento, alcanzan el promedio normal al ano de edad corregida. Los $\mathrm{RN}$ de menos de $1.000 \mathrm{~g}$ no logran esta meta $y$, según algunos ${ }^{12}$, el $37 \%$ tiene una talla y el 17\% un diámetro cefálico bajo el percentil 3 al cumplir un año de edad postconcepcional.

\section{RESUMEN}

Se estudia en forma prospectiva, hasta el año de edad, el crecimiento longitudinal de 154 RN de peso inferior a $1.501 \mathrm{~g}$ atendidos en el Hospital Roberto del Río entre 1983 y 1987. Para el análisis se dividen los sujetos en tres grupos: el grupo $A$ incluye $44 \mathrm{RN}$ de peso inferior a 1.001 $\mathrm{g}$; el grupo B, 68 de 1.000 a $1.250 \mathrm{~g}, \mathrm{y}$ el grupo C, 42 de 1.251 a $1.500 \mathrm{~g}$.

Para comparar los resultados se usan las curvas de crecimiento de Patrí y colaboradores de ninfos sanos con un peso promedio de nacimiento de $3.318 \mathrm{~g}$, pertenecientes al mismo medio socioeconómico y área geográfica.

Los RN del grupo A pesan, al año, $6.724 \pm$ $804 \mathrm{~g}$, los del grupo B, $7.782 \pm 927 \mathrm{~g}$, y los del grupo C, $7.941 \pm 903$ g. Estos valores, lo mismo que los de la talla, están bajo $2 \mathrm{DE}$ del promedio normal, en los nifros del grupo A. Entre 1 y 2 DS en los de los grupos B y C. El perímetro cefáli. co, al año de edad postnatal, se acerca más a los canales normales en los grupos B y C. Sólo los de peso inferior a $1.000 \mathrm{~g}$ tienen una circunferencia craneana bajo $2 \mathrm{DE}$ del promedio normal.

Aparentemente el crecimiento postnatal no logra reproducir el del feto in utero. Al año de edad el peso promedio de todo el grupo está muy por debajo del esperado para un niño nacido a término. La talla y, sobre todo, el perímetro cefálico se acercan más a lo considerado como normal. Las tablas de crecimiento propuestas para niños de menos de 1.501 g podrian permitir determinar el peso, la talia, el perimetro cefálico promedio y el mínimo aceptable correspondiente a 1 DE para cualquier edad postnatal cuando sólo se conoce el peso de nacimiento. Es necesario seguir estudiando en forma colaborativa el crecimiento "típico" del ENMBP que sobrevive en las actuales tecnologias.

\section{AGRADECIMIENTOS}

Agradecemos la colaboración de la señorita María Angélica Villarroel, quien está a cargo del Consultorio Externo de Seguimiento del RNMBP.

\section{REFERENCIAS}

1. Gairdner D., Pearson I: A growth chart for premature and others infants. Arch Dis Child 1971; 46: 783-787.

2. Maisels $M$.: Growth chart for sick premature infants. J Pediatr 1981;98: 663-665.

3. Babson S.: Growth of low birth-weight infants. J Pediatr 1970; 77: 11-15.

4. Gill A., Yu V., Bajuk B., A stbury J.: Postnatal growth in infants born before 30 weeks gestation. Arch Dis Child 1986; 61: 549-553.

5. Kimble K., Ariagno R., Stevenson D., Sunshine $P h .:$ Growth to age 3 yars among very low weight 
scauelae-free survivors of modern neonatal intensive care. J Pediat 1982; 100: 622-624.

6. Ross G., Krauss A., Auld P.: Growth achievement in low birth weight premature infants: Relation. ship to neurobehavioral outcome at one year. $J$ Pediatr 1983; 103: 105-108.

7. Hodgson M., Rath B., Brunnet A., Lauy $R$. Torres $J$.: Alimentación de RN de muy bajo peso con leche de su propia madre. Rev Chil Pediatr $1987 ; 58: 296-301$.

8. Brositus K., Ritter D., Kenny J.: Postnatal growth curve of the infant with extremely low birth weight who was fed enteraty. Pediatrics 1984; 74 : 778 782.

9. Gibbs J.: Routine total and supplemental parenteral mutrition for the very low birth weight infant. Ross Conference on Pediatric Research, 1980.
10. Rizzardini M., Ferreiro M., Nilo P., Palominos M., Pantoja S.: Crecimiento postnatal del recién nacido de muy bajo peso: [ Crecimiento inmediato. Rev Chil Pediatr 1988; 59: 166-169.

11. Patri S., Sepúlveda $H$., Valenzuela $C$ : Antropometría del niño chileno de 0 a 6 años. Editorial Andrés Bello, Santiago-Chile, 1984.

12. Britton $S$, Chin B., Fitzhardinge $P .:$ Is intessive care justified for infants weighting less than $801 \mathrm{~g}$ at birth? J Pediatr 1981;99: 937-941.

13. Weldt E., Hering $E$., Valenzuela B., Angulo $Y_{\text {., }}$ Neira $A$.: Seguimiento de niños con peso de nacimiento inferior a 1.500 g. Rev Chil Pediatr $1986 ; 57: 51-56$.

14. Gross S., Dehler J., Eckerman C.: Head growth and developmental out come in very low birth weight infants. Pediatrics 1983;71:70-76. 